\title{
A GRID OF CHEMICAL EVOLUTION MODELS ALONG THE HUBBLE SEQUENCE
}

\author{
M. MOLLÁ AND A. I. DíAZ \\ Universidad Autónoma de Madrid, 28049 Cantoblanco, Spain \\ E-mail: mercedes@pollux.ft.uam,angeles@pollux.ft.uam.es \\ AND F. FERRINI \\ Università di Pisa, Piazza Torricelli 2, 56100 Pisa, Italy \\ E-mail: federico@astro2.difi.unipi.it
}

\begin{abstract}
We have computed a grid of multiphase chemical evolution models whose results are valid for any spiral galaxy, using as input the maximum rotation velocity and the morphological type or index $\mathrm{T}$
\end{abstract}

\section{Introduction}

In previous works, we have calculated the chemical evolution of the Solar Neighborhood (Ferrini et al 1 ), the Galactic Disc and Bulge (Ferrini et al 1 , (4), and a sample of spiral galaxies (Mollá et al 5 ) with the multiphase model. All of them reproduce with success the observational data, in particular those related with the disk radial distributions. They also predict the correlations found along the Hubble Sequence (Mollá \& Roy computed models is small to ensure it. Now, we have computed an extended grid of multiphase models, which allow the comparison of observations with theoretical models for a large range of galaxy masses and morphological types.

\section{The Multiphase Model Description}

We assume a spherical protogalaxy with a gas mass which collapses to fall onto the equatorial plane by forming the disk as a secondary structure. The sphere is divided up into concentric cylindrical regions $1 \mathrm{kpc}$ wide, with a halo and a disk region. The Universal Rotation Curve from Persic, Salucci \& Steel $\mathrm{B}$, an analytical expression for $\mathrm{V}(\mathrm{R})$, is used to calculate the radial distributions of the mass included in each of our cylinders $\Delta \mathrm{M}(\mathrm{R})$.

The characteristic gas infall rate from the halo to the disk is proportional to a parameter $f$, which, in turn, is inversely proportional to the collapse timescale $\tau_{0}$, dependent on the total mass (Gallagher et al S) $^{3}$. It is easily computed from its value for the MWG model, $\tau \odot$, and the ratio between total masses: $\tau_{0}=\tau_{\odot}\left(M_{9}, \mathrm{gal} / M_{9, M W G}\right)^{-1 / 2}$. 
Stars form out in the halo, by a Schmidt law with a proportionality factor $K$. In the disk, stars form in two steps: molecular clouds form from the diffuse gas with a proportionality factor $\mu$; then cloud-cloud collisions produce stars by a spontaneous process, at a rate proportional to a parameter $H$. Moreover a stimulated star formation process, by the interaction of massive stars with surrounding molecular cloud, is assumed,s proportional to a parameter $a$,

These parameters are variable along the radius, by the volume effect, through the use of the process efficiencies $(\epsilon$ 's), valid for the whole galaxy. We assume that the two efficiencies $\epsilon_{K}$ and $\epsilon_{a}$ are constant for all halos and galaxies, while $\epsilon_{\mu}$, and $\epsilon_{H}$ change according the Hubble type T by a function $\epsilon=\exp \left(-T^{2} / a\right)$. We have computed 10 types of galaxies for each mass.

\section{Preliminary Results and Conclusions}

A biparametric grid of models has been obtained for types 1 to 10 and different luminosities. We obtain radial distributions for the star formation rate, the diffuse and molecular gas densities and the elemental abundances.

The most important result concerns the oxygen abundances: the radial gradient only appears for the intermediate types $(7<\mathrm{T}<4)$ at all galaxy masses, being larger for the less massive galaxies. However, the latest ones $(\mathrm{T} \geq 8)$ have not developed a gradient in a Hubble time, their abundances being $12+\log (O / H) \sim 7.5-8$. The early types $\mathrm{T} \leq 5$ reach a saturation level, flattening the gradient once again, after having a steep one early times.

This important result reproduces the observations and it solves the apparent inconsistency of the largest gradients appearing in late type spirals, while some irregulars shows no gradient at all. The explanation resides in the stimulated star formation which, being a local process, maintains a minimum level of star formation constant for all regions.

\section{References}

1. Ferrini, F., Matteucci, F., Pardi, C., \& Penco, U., ApJ 387, 138 (1992).

2. Ferrini, F., Mollá, M., Pardi, C., \& Díaz, A. I, ApJ 427, 745 (1994).

3. Gallagher, J. S., Hunter, D. A., \& Tutukov, A. V., ApJ 284, 544 (1984)..

4. Mollá, M. \& Ferrini, F. ApJ 454, 726 (1995)

5. Mollá, M., Ferrini, F., \& Díaz, A. I,ApJ 466, 668 (1996).

6. Mollá, M., Hardy, E., \& Beauchamp, D., ApJ 513, 695 (1999).

7. Mollá, M. \& Roy, J.-R., in Chemical Evolution from zero to high redshift, eds. J.R.Walsh \& M.R. Rosa (Springer-Verlag, Berlin, 1999)

8. Persic, M., Salucci, P. \& Steel, F., MNRAS 281, 27 (1996).

molla3: submitted to World Scientific on July 24, 2021 